\title{
The Turbutron
}

\author{
HOWARD E. BRANDT
}

\begin{abstract}
The physics of the turbutron is reviewed. This newly proposed high-power millimeter-wave source consists of an intensely oscillating relativistic turbulent electron plasma created in and beyond the gap of a diode configuration. The diode consists of an explosive cathode emitter and an extended anode structure connected to the inner and outer conductors, respectively, of a high-voltage pulse line under conditions of space-charge saturation. The gap spacing determines the dominant mode of the turbulent longitudinal waves which are directly converted into free transverse waves polarized parallel to the electron beam. The applied voltage waveform, cathode diameter, total scalar potential, nonlinear bunching mechanisms, and virtual-cathode dynamics determine the complex spectral characteristics. For a turbutron with a megavolt pulse across a 3-mm gap and without a resonator, calculations predict the immediate feasibility of gigawatt power levels at 35 GHz.
\end{abstract}

\section{INTRODUCTION}

$\mathrm{T}$ HE TURBUTRON is a new concept for a high-power gigawatt level source of millimeter-wave radiation [1]. The experimental prototype will be capable of producing gigawatt power levels at $35 \mathrm{GHz}$. This paper reviews the physics and design of the turbutron and presents results of calculations of the electron dynamics and radiated millimeter-wave spectrum for the prototype.

In Section II, previous and current related works on reflex triodes, reflex diodes, vircators, and other virtualcathode microwave sources are briefly mentioned. Section III reviews the theory and design of the turbutron prototype. Section IV presents the results of calculations of the electron density distribution and the radiated millimeterwave power spectrum. Section $\mathrm{V}$ addresses the nature of the relativistic turbulent electron plasma in the turbutron. In Section VI, the physical characteristics of the virtual cathode are discussed. Section VII presents methods for tailoring the turbutron spectrum to requirements. Section VIII draws conclusions.

\section{Background and Related Work}

Considerable interest exists presently in the design of virtual-cathode microwave generators, including such devices as reflex triodes, reflex diodes, vircators, and the newly proposed turbutron. Each of these devices has a boundary beyond the primary anode plane through which relatively few electrons penetrate and from which most electrons are reflected.

Some years ago at Harry Diamond Laboratories (HDL), the Naval Research Laboratory, and in the Soviet Union,

Manuscript received March 7, 1985; revised July 18, 1985.

The author is with the U.S. Army Electronics Research and Development Command, Harry Diamond Laboratories, Adelphi, MD 20783-1197. the reflex triode was experimentally demonstrated to be an impressive high-power source of microwave radiation [2]-[4]. In particular, at HDL 10-ns bursts of gigawattlevel microwave radiation were achieved in $X$ band at 10 $\mathrm{GHz}$ [2], [5]-[13]. In the reflex triode the cathode and plate are at ground potential and the anodal grid is at a high megavolt level voltage. An intensely oscillating relativistic electron plasma is created in and beyond the accelerating gap and is the source of the microwaves. Most of the electrons are reflected at the virtual cathode, the oscillating boundary beyond which few electrons penetrate. It has been demonstrated that diode and triode configurations do not differ significantly in their dynamical and radiation characteristics [1]. In particular, a diode configuration may be modeled analogously to the triode [2], but with the plate and grid at equal positive megavolt potential with respect to the cathode. In the case of the diode, a virtual cathode forms at nearly the same position as for the triode. In both devices the electrons are concentrated within $\sim 1 \mathrm{~cm}$ of both sides of the grid for a $1-\mathrm{cm}$ gap, and the dominant spectral characteristics are comparable. Both the level and the frequency content of the power spectral density are very similar. Calculations show that if the HDL reflex triode experiment were repeated with the same megavolt pulse across the centimeter gap of a diode configuration, gigawatt power levels in $X$ band would again be obtained.

The newly proposed turbutron has such a diode configuration but with a 3-mm gap instead of a $1-\mathrm{cm}$ gap in order to increase the dominant frequency from 10 to $35 \mathrm{GHz}$. The previous success of the reflex triode experiment and of the theory [2] in predicting gigawatt power levels at 10 $\mathrm{GHz}$, together with the conclusion that diode and triode configurations have comparable microwave characteristics, provides a strong logical argument for the viability of the turbutron concept.

The popular vircator [14]-[21] is essentially a reflex diode in which the reflexing electrons are removed in some way, for example, by shaping the confining magnetic field or employing a slotted anode. The remaining electrons are concentrated in a potential well localized at the virtual cathode and oscillating in a single mode. The operation of the vircator typically requires the frequency of the oscillating virtual cathode to be the same as that of a mode of an axial waveguide/drift tube to which it is resonantly coupled. The turbutron, however, will produce gigawatt radiation without the requirements of a tuned axial waveguide or magnetic focusing of the beam, and without wasting a significant fraction of the electron beam. The tur- 
butron is not as complicated as the vircator nor does it require the precision tuning of the vircator. In the vircator the radiation is typically extracted from the end of the axial waveguide, whereas in the turbutron the radiation is extracted directly transverse to the beam. In addition, the turbutron diode configuration results in an increase of the dominant frequency to $35 \mathrm{GHz}$ while still maintaining gigawatt power levels, thereby offering a significant improvement over high-power reflex triodes, diodes, and vircators which typically operate in $X$ band.

\section{Theory and Design of the Turbutron}

The turbutron consists of an intensely oscillating relativistic turbulent electron plasma created in and beyond the gap of a diode configuration. The prototype diode shown in Fig. 1 consists of an explosive carbon cathode emitter and an extended cylindrical anode structure connected to the inner and outer conductors, respectively, of a megavolt voltage pulse line under conditions of charge saturation. For the turbutron to dominantly produce 35 $\mathrm{GHz}$, the gap is $3 \mathrm{~mm}$. In the first-generation turbutron, the anode structure acts only to create an equipotential surface that is at ground relative to the cathode, which is at a negative megavolt potential. The cathode for the prototype is taken to be $5.1 \mathrm{~cm}$ in diameter, thereby producing a 5.1-cm-diameter solid cylindrical beam. Increasing the gap will increase the dominant wavelength. Gaps smaller than $3 \mathrm{~mm}$ are limited by gap closure. The primary anode surface will initially consist of $6-\mu \mathrm{m}$ aluminized Mylar foil, as was used in the HDL reflex triode. Eventual designs will explore the feasibility of a wire grid or preferably a circular hole enabling repetitive operation. An external magnetic field of $\sim 1 \mathrm{~T}$ directed along the turbutron axis will confine the electron plasma radially. The vacuum is to be $\sim 10^{-4}$ torr.

When the turbutron is fired, a voltage pulse of $\sim 1 \mathrm{MV}$ will move down the pulse line and across the cathodeanode gap, explosively pulling tens of kiloamperes of average current of electrons from the cathode. The gap and beyond will fill with a turbulent electron plasma having a broad turbulent spectrum of modes of oscillation but peaking at $35 \mathrm{GHz}$ and thereby producing tens of nanoseconds of pulsed high-power millimeter waves radiating out transverse to the beam and polarized parallel to the axis of the turbutron. A reflector antenna, located coaxially with the cylindrical anode structure and externally to the magnet coils, can also be utilized to reflect the radiation out parallel and external to the diode axis and produce a prescribed gain pattern. The device is called a turbutron to emphasize the fact that the source of radiation is the turbulent state of the relativistic electron plasma.

To calculate the dynamics of the turbulent electron plasma in the turbutron, a one-dimensional space- and time-dependent computer code based on a relativistic sheet model was written directly analogous to that of the triode [2] but modified so that the plate and grid potentials are equal and positive with respect to the cathode. It is to be

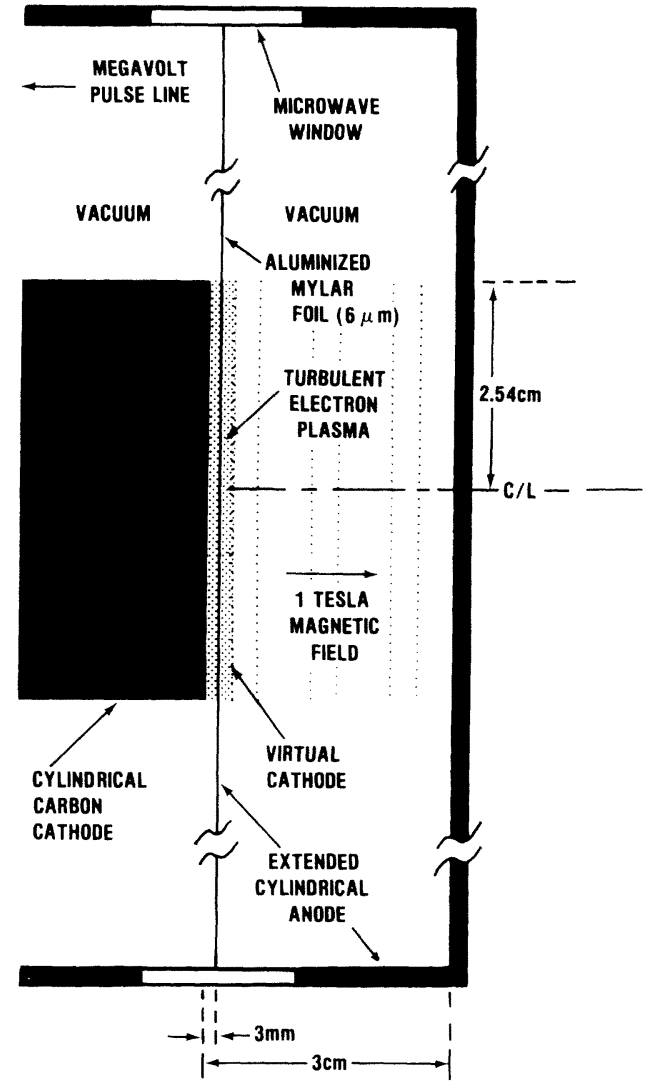

Fig. 1. Turbutron geometry (magnet coils and coaxial reflector-antenna system not shown).

stressed that for the triode a sheet model successfully predicted power levels within a factor of two and frequency nearly exactly. Also it successfully calculated the formation of the turbulent state of the electron plasma, transient bunching, the oscillating virtual-cathode dynamics, shocklike discontinuities in the electric field, and acceleration of electron bunches through the virtual cathode and out to the plate with energies far exceeding the applied potential [2]. In the past, analogous sheet models have been used extensively to model plasma and diode electron dynamics [22]-[28].

Thus, to describe the essential underlying electron dynamics in the turbutron, the real three-dimensional timedependent configuration is approximated by a one-dimensional time-dependent configuration. In support of this is the fact that the applied magnetic field confines the beam in the radial direction. In the limit of an extremely strong magnetic field, the electron motion would be confined to the magnetic-field lines. It is assumed that the dominant force is that due to the high applied voltage so that, to first order, the electron motion is predominantly parallel to the turbutron axis. One would also expect the one-dimensional model to be suitable for the turbutron because of its large ratio of cathode diameter to cathode-anode gap.

The cathode plane, located at $x=0$, is taken to be at zero electrical potential. The anode foil and far wall of the extended anode structure are located at $x=d_{1}$ and $x=$ $d_{2}$ and are at the potential of the prescribed voltage pulse.

The relativistic turbulent electron plasma within the 
diode region is represented by many sheets. Thus the unsmoothed electron space-charge density $\rho(x, t)$, at any instant $t$ as a function of distance $x$ from the emission cathode plane, is represented by

$$
\rho(x, t)=\lim _{M(t) \rightarrow \infty} \sigma_{M} \sum_{m=1}^{M(t)} \delta\left(x-x_{m}(t)\right) .
$$

Here, $M(t)$ is the number of sheets in the diode region at time $t, \sigma_{M}$ is the charge density of each sheet and is taken to be the same for each sheet, $\delta$ is the one-dimensional Dirac delta function, and $x_{m}(t)$ is the position of the $m$ th sheet at time $t$. In practice, the infinite limit in (1) must clearly be replaced by a finite one. The time-average number of sheets in the diode region $\bar{M}$ should be chosen as large as simulation cost permits. The dynamics will control coordinates $x_{m}(t)$ and spacing of the constant and equalcharge density sheets so that regions of high-charge density will have a high density of sheets.

A primary theoretical weakness of the sheet model is that, in practice, each sheet will correspond to $\sim 10^{10}$ electrons, whose separate degrees of freedom are ignored and which are effectively constrained to move bound together as a whole. This same fundamental criticism holds also for particle-in-cell models and, for that matter, for any "fluid element" representation. For the model at hand, this problem is less serious, the larger the choice of $\bar{M}$. The final justification for the sufficient largeness of $\bar{M}$ is the convergence of quantities of interest. However, in practice, establishing such convergence is prohibited by cost and the difficulty of even quasi-analytical proof.

In the simple model presented here, ion formation at the anode and the resulting ionic component of the charge distribution are ignored on the grounds that significant ion displacement will be relatively delayed because of the large ion-to-electron mass ratio.

The electric field $E\left(x_{n}, t\right)$ at location $x_{n}$ of the $n$th sheet at time $t$, due to both the externally applied voltage $V(t)$ and the space-charge fields arising from all the other sheets, is determined by solving Poisson's equation, which results in

$$
E\left(x_{n}, t\right)=\left\{\begin{array}{c}
\frac{\sigma}{\epsilon_{0}}\left\{-\left[M_{1}(t)-M_{11}\left(x_{n}(t)\right)\right]\right. \\
\left.+\frac{1}{2}+M_{1}(t) \frac{\bar{x}_{1}(t)}{d_{1}}\right\}-\frac{V(t)}{d_{1}}, \\
0<x_{n} \leq d_{1} \\
\frac{\sigma}{\epsilon_{0}}\left\{-\left[M_{2}(t)-M_{21}\left(x_{n}(t)\right)\right]\right. \\
\left.+\frac{1}{2}+M_{2}(t) \frac{\bar{x}_{2}(t)-d_{1}}{d_{2}-d_{1}}\right\}, \\
d_{1}<x_{n}<d_{2} .
\end{array}\right.
$$

The upper expression holds at a sheet lying on the emission cathode side of the anode foil, and the lower expression on the other side. Here $d_{1}$ is the cathode-anode gap; $d_{2}$ is the separation between the cathode and the far wall; $\sigma$ is the sheet charge density; $\epsilon_{0}$ is the permittivity of free space; $M_{1}(t)$ and $M_{2}(t)$ are the number of sheets on the cathode side and other side of the anode, respectively, at time $t ; M_{11}\left(x_{n}(t)\right)$ is the number of sheets between the cathode and the $n$th sheet when it is on the cathode side of the anode; $M_{21}\left(x_{n}(t)\right)$ is the number of sheets between the anode foil and the $n$th sheet when it is on the other side; and $\bar{x}_{1}(t)$ and $\bar{x}_{2}(t)$ are the mean separations from the cathode for all sheets on the cathode and other sides, respectively, of the anode foil.

The time step for the model is taken to be a small fraction of a cathode-anode light transit time. Since the electrons are limited by relativity to velocities less than the velocity of light, this choice of time step should provide sufficient time resolution of the electron dynamics. Thus the time step $\Delta t$ is given by

$$
\Delta t=d_{1} / N c
$$

where $N$ is some large number and $c$ is the speed of light.

Sheet electron momenta $p_{n}(t+\Delta t)$ and positions $x_{n}$ $(t+\Delta t)$ at time $t+\Delta t$ are then easily expressed in terms of those at time $t$ by use of the Lorentz force equation, by which one obtains

$$
\begin{aligned}
p_{n}(t+\Delta t)= & p_{n}(t)+e E\left(x_{n}, t\right) \Delta t \\
x_{n}(t+\Delta t)= & x_{n}(t)+\frac{1}{2}\left[1+\left(\frac{p_{n}(t)}{m c}\right)^{2}\right]^{-3 / 2} \\
& \times\left\{\left[1+2\left(\frac{p_{n}(t)}{m c}\right)^{2}\right] \frac{p_{n}(t)}{m c}\right. \\
& \left.+\frac{p_{n}(t+\Delta t)}{m c}\right\} c \Delta t .
\end{aligned}
$$

Index $n$ corresponds to the $n$th sheet, and $e$ and $m$ are the charge and the mass of the electron, respectively. A sheet is allowed to enter the gap only at those time steps for which the total electric field points into the emission cathode. A sheet is taken to leave the system whenever it hits the far wall or returns to the emission cathode. A sheet is also subtracted whenever the energy that it loses in passing through the anode foil reduces its kinetic energy to zero. Thus the number of sheets $M(t+\Delta t)$ at time $t+\Delta t$ is given in terms of the number of sheets at time $t$ by

$$
\begin{aligned}
M(t+ & \Delta t) \\
= & M(t)+\theta(-E(0, t))-\sum_{m=1}^{M(t)}\left[\theta\left(d_{2}-x_{m}(t)\right)\right. \\
& \cdot \theta\left(x_{m}(t+\Delta t)-d_{2}\right)+\theta\left(x_{m}(t)\right) \theta\left(-x_{m}(t+\Delta t)\right) \\
& +\theta\left(d_{1}-x_{m}(t)\right) \theta\left(x_{m}(t+\Delta t)-d_{1}\right) \\
& \cdot \theta\left(\Delta p_{m}-p_{m}(t+\Delta t)\right)+\theta\left(x_{m}(t)-d_{1}\right) \\
& \left.\cdot \theta\left(d_{1}-x_{m}(t+\Delta t)\right) \theta\left(\Delta p_{m}+p_{m}(t+\Delta t)\right)\right] . \quad(6)
\end{aligned}
$$


Here, $\theta$ is the theta function, $E(0, t)$ is the total electric field at the emission cathode, and $\Delta p_{m}$ is the momentum loss experienced by the $m$ th sheet in passing through the anode foil as determined by ionization and radiative loss tables.

The surface charge density $\sigma$ of the sheets is determined by the following considerations. The charge in the diode region will build up to the point where it can contain no more; namely, further injection of charge will result in charge ejection. Under such a condition of space-charge saturation, the total time-averaged charge $\bar{Q}$ in the diode region is proportional to the applied voltage $V$, namely

$$
\bar{Q}=K V
$$

where $K$ is the space-charge capacity of the turbutron [2], [6]. It is independent of applied voltage and is determined only by the turbutron geometry.

The time-averaged charge may be expressed in terms of the time-averaged number of sheets $\bar{M}$ by

$$
\bar{Q}=A \sigma \bar{M}
$$

where $A$ is the cross-sectional area of the beam, which is assumed equal to the cathode area, and $\sigma$ is the sheet surface charge density. By combining (7) and (8), the required sheet surface charge density is given by

$$
\sigma=\frac{K V}{\bar{M} A} \text {. }
$$

Therefore, in practice, one chooses the averaged number of sheets to be some large, but affordable number to faithfully represent the space-charge distribution: the space-charge capacity is self-consistently calculated under conditions of space-charge saturation; the voltage and the beam area are given; and the sheet surface charge density is thereby determined. Equations (1)-(9) form the algebraic basis for the numerical simulation of the nonlinear electron plasma dynamics in the turbutron.

It is assumed here that the real three-dimensional beam dynamics may be approximated by the one-dimensional model. For the cylindrical beam of radius $R$, the average power spectral density per steradian $\left\langle d^{2} P_{\perp} / d \omega d \Omega\right\rangle$ radiated perpendicular to the beam can be shown to be given by

$$
\left\langle\frac{d^{2} P_{\perp}}{d \omega d \Omega}\right\rangle=\frac{\mu_{0} \pi \sigma^{2} R^{4}}{4 c T} \omega^{2}|\Sigma(\omega)|^{2} F^{2}(\omega)
$$

where

$$
\Sigma(\omega)=\frac{1}{2 \pi} \int_{-\infty}^{\infty} d t e^{-i \omega t} \sum_{m=1}^{M(t)} v_{m}(t)
$$

and

$$
F(\omega)=\frac{2 J_{1}(\omega R / c)}{\omega R / c}
$$

Here, $d \Omega$ is the differential solid angle; $\mu_{0}$ is the permeability of free space; $\sigma$ is the sheet surface charge density; $R$ is the beam radius; $c$ is the speed of light; $T$ is the volt- age pulse duration; $\omega$ is the angular frequency; $M(t)$ is the number of sheets at time $t ; v_{m}(t)$ is the velocity of the $m$ th sheet at time $t$; and $J_{1}$ is the first-order Bessel function of the first kind. Equation (10) is obtained by solving the wave equation with a current distribution represented by the calculated sheet dynamics, including radiative time delays only perpendicular to the beam, and by using the ordinary dipole far-field approximation. The quantity $\Sigma(\omega)$ is the time Fourier transform of the sum of the sheet velocities. The quantity $F(\omega)$ is a geometric interference form factor resulting from the relative time delays across the beam cross section.

Although the model ignores possible three-dimensional effects-in particular, those due to magnetic fields-and employs a coarse grained sheet model representation, it is expected to successfully characterize the main features of the radiated millimeter-wave spectrum for the turbutron.

\section{Electron Distribution and Millimeter- Wave Spectrum}

The calculated electron charge density distribution as a function of time is shown in Fig. 2. Here the electron charge density in coulombs per cubic meter is plotted as a function of distance (in millimeters) from the cathode and time (in picoseconds) from the initiation of a constant 1MV applied voltage across the 3-mm gap. The far wall of the extended anode structure is $3 \mathrm{~cm}$ from the cathode. Only the first 250 ps of the time history and the first 20 $\mathrm{mm}$ from the cathode are shown in Fig. 2. Important characteristics of the turbulent electron plasma are discussed in Section V.

Equation (10) was used to calculate the average transverse power spectral density that is radiated out transverse to the beam and in the far field. The spectrum is broad band, as is characteristic of a turbulent source, but peaks in the millimeter-wave region of the spectrum. In Fig. 3 the average transverse power spectral density in megawatts per steradian-gigahertz is plotted as a function of frequency in the millimeter band from 25 to $50 \mathrm{GHz}$, again for $1 \mathrm{MV}$ across a 3-mm gap. It is seen to peak near 35 $\mathrm{GHz}$ with an average power of $30 \mathrm{MW} / \mathrm{sr} \cdot \mathrm{GHz}$ over a band $\sim 3 \mathrm{GHz}$ wide. As was the case for the triode [2], this level is consistent with gigawatt-level peak power for the turbutron. The radiated power from the turbutron at $35 \mathrm{GHz}$ for a 1-MV gap voltage is about the same as that for the triode at $10 \mathrm{GHz}$ for a 2-MV gap voltage [2]. It is to be noted also that the interference form factor [2] for the turbutron with the $5.1-\mathrm{cm}$ beam diameter peaks near the dominant mode at $35 \mathrm{GHz}$. Also, as was shown for the triode, a nonconstant voltage pulse will introduce additional fine structure to the spectrum but will not significantly alter the main spectral features.

\section{The Turbulent Electron Plasma}

The numerical calculations based on the sheet model reveal many important characteristics of the turbulent relativistic electron plasma in the turbutron. At the onset of 


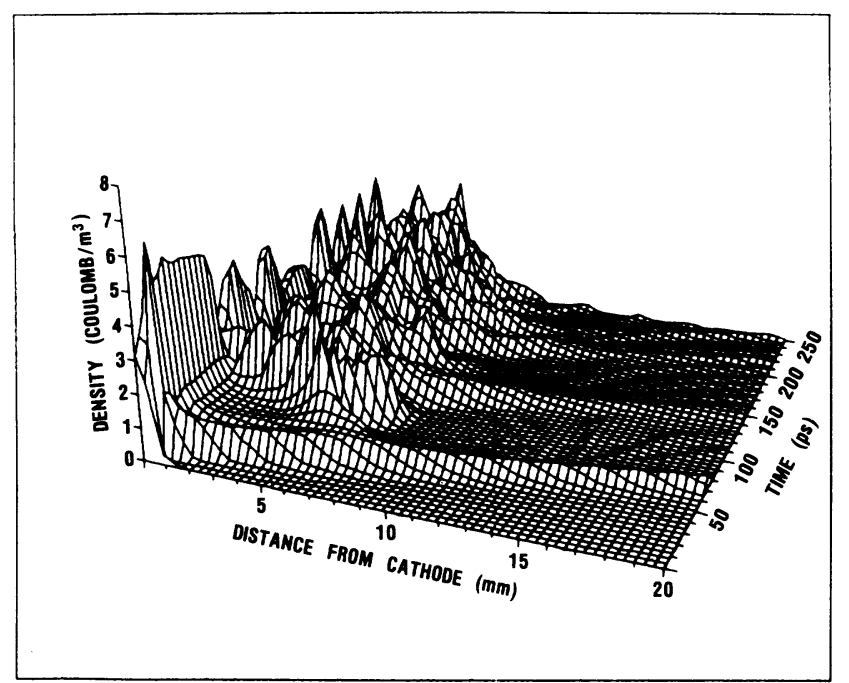

Fig. 2. Electron charge density for the turbutron as a function of distance from cathode and time. Primary anode plane is $3 \mathrm{~mm}$ from cathode, far anodal plane is at $30 \mathrm{~mm}$, and beam diameter is $5.1 \mathrm{~cm}$. Applied voltage is $1 \mathrm{MV}$.

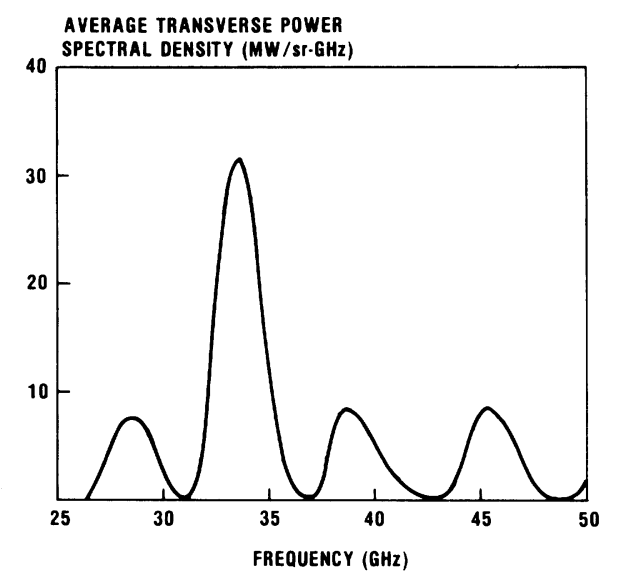

Fig. 3. Turbutron millimeter-wave spectrum perpendicular to beam for $1 \mathrm{MV}$ across a 3-mm gap and a 5.1-cm-diameter beam.

the voltage pulse across the 3-mm cathode-anode gap, electrons are explosively pulled out of the cathode plasma and begin to fill up the diode region. As may be seen from Fig. 2, during the first 60 ps there is a smooth flow of the electrons toward the far wall of the anode at $\sim 0.9$ velocity of light. The initial electron density wave has a charge density in the leading edge of $\sim 1 \mathrm{C} / \mathrm{m}^{3}$. After $\sim 60 \mathrm{ps}$ one sees the onset of space-charge limiting, the formation of a virtual cathode, and the onset of the turbulent state of the electron plasma between the emission and virtual cathodes. The virtual cathode in the turbutron resembles that in the reflex triode [2]-namely, it is an oscillating boundary which forms within the turbutron extended-anode structure at a time-average distance from the cathode of approximately $6 \mathrm{~mm}$, or twice the cathode-anode gap. In Fig. 4 is plotted the first 125 ps of the time history in Fig. 2 , with an expanded scale to display the virtual-cathode transition in greater detail.

Calculations of the total scalar potential show that the virtual cathode is also the location of a discontinuity in the electric field. The possible interpretation of the dis-

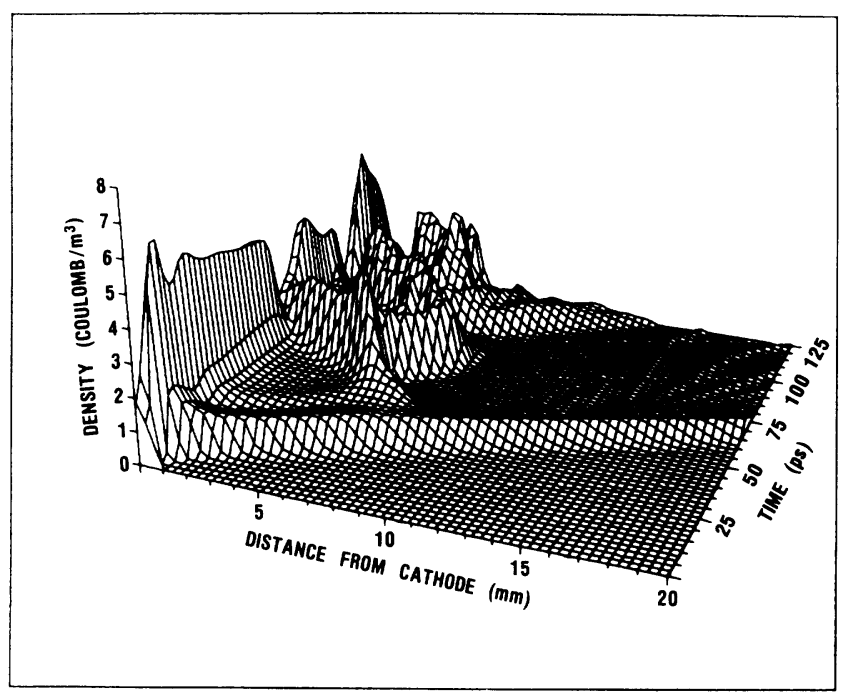

Fig. 4. Electron charge density of Fig. 2 for first 125 ps and including $20 \mathrm{~mm}$ of turbutron axis.

continuity as an oscillatory collisionless shock front is under investigation. A possible alternative framework for investigating such shocks would be to find collisionless shock solutions to the Vlasov-Maxwell model [1] of the turbutron, characterized by discontinuities in the electric field and highly bunched electron distributions. The sheet model calculations also show that some relativistic electron density wave fronts penetrate the virtual cathode and move at near-constant high velocity toward the far wall. The possibility that these are also electrodynamic shock waves is also being investigated. The particles in these waves are accelerated to energies much greater than the 1MV accelerating potential [2], [6], [7].

Following the formation of the virtual cathode, the total space charge saturates with oscillations about the time average. As can be seen in Fig. 2, the dynamical spectral distribution of the electron plasma is very broad band with many modes highly excited. This is the turbulent state of the pure electron plasma [29]. Electrons entering the gap with favorable phases relative to a given frequency component of the resultant space-charge fields give up energy to the fields and remain in the diode region, while those with unfavorable phases gain energy from the fields and are ejected from the turbutron. Favorably phased electrons tend to be grouped together spatially since they enter at about the same time. Also, space-charge limiting periodically limits the subsequent entrance of electrons at the emission cathode, as may be seen from the quasi-periodic peaks of $\sim 5 \mathrm{C} / \mathrm{m}^{3}$ in the electron density at the cathode. It is noteworthy that the frequency of this quasi-periodic space-charge limiting is near the dominant frequency of emission at $35 \mathrm{GHz}$. The primary mode of oscillation of the virtual cathode is also near the dominant emission frequency, as in the case of the triode [2]. The phase-selection mechanism gives rise to electron bunching. While the electron bunches oscillate back and forth about the anode, they also interact with one another, scattering, ejecting, and capturing electrons from one another and thereby being either depleted or augmented in size. Bunches are 
born and bunches die. This is the regime of strong turbulence of the relativistic electron plasma.

Insight into the transient bunching might also be gained by attempting to find approximate lump or soliton-like states of the highly nonlinear Vlasov-Maxwell model of the turbutron [1]. The complete turbulent state should be equally well characterized by the Vlasov-Maxwell equations as by the sheet model, and this is currently being investigated. It is important that boundary conditions analogous to those in the sheet model [2] be incorporated in the Vlasov description. Electrons enter the diode region only when the total electric field at the cathode is negative. They are lost when they hit the cathode or the far wall of the anode structure, or when their energy loss in passing through the anode foil equals their incident energy. A control parameter for bifurcations is likely to be the number of electrons entering per unit time, when they enter.

It is also timely to mention that the nonlinear dynamical iterations at each time step in the sheet model, (2), (4), (5), are analogous to the iterative maps of chaotic dynamics [30]-[33], which results in the chaotic state. Likely connections between chaos and turbulence in relativistic electron plasmas should be explored. Turbulence does not preclude order.

The broad-band dynamical spectrum of the turbulent electron plasma with the associated high-frequency oscillations of the transient electron bunches result in a broadband microwave spectrum. Noteworthy is the fact that the total power radiated in the prototype turbutron is $\sim 1 \mathrm{GW} /$ sr, with significant broad-band emission also in $S, C, X$, $K_{u}$, and $K$ band, in addition to the dominant millimeterwave band shown in Fig. 3. The turbulent longitudinal waves are directly converted into free transverse waves polarized parallel to the turbutron axis.

It is important to gain increased theoretical and experimental understanding of the nonlinear states of the relativistic pure electron plasma since they determine the radiated microwave spectrum in the turbutron and other high-voltage and high-current virtual-cathode devices.

\section{Vi. The Virtual Cathode}

It is good to point out significant differences between the concept of a virtual cathode in the turbutron and the triode compared to other so-called virtual-cathode microwave generators. In the turbutron and triode the virtual cathode is oscillatory both in position and potential and at its location there is a discontinuity in the slope of the scalar potential or, equivalently, a jump in the electric field [1], [2]. It can also be characterized as a boundary from which most electrons are reflected and through which few electrons penetrate. The bulk of the electrons are spread out turbulently between the emission and virtual cathodes and are not concentrated at the virtual cathode as is contemplated for the vircator. The virtual cathode is merely one, albeit very visible, feature of the nonlinear state of the electron plasma in the turbutron. It must be stressed that many other characteristics of the plasma, such as its dynamical spectral distribution, coherence characteris- tics, and relative phase of the bunches, are also important in determining the properties of the radiated power spectrum.

\section{Spectral Design}

For the solid cylindrical beam in the prototype turbutron, the interference form factor is given by (12). Zeros in the spectrum occur for frequency $f$ such that $J_{1}(2 \pi f R /$ $c$ ) in the form factor is vanishing, where $J_{1}$ is a first-order Bessel function of the first kind, $R$ is the beam radius, and $c$ is the speed of light. In the case of the prototype with a 5.1-cm beam diameter, this factor determines the zeros at 25, 31, 37, 43, and $49 \mathrm{GHz}$ in Fig. 3. Varying the radius will cause the transverse power spectral density to vary as $\left[R J_{1}(2 \pi f R / c)\right]^{2}$, and the zeros resulting from destructive interference can be moved accordingly [2]. Of course, changing the beam geometry, making it hollow, for example, allows one also to change the expression for the form factor and the associated spectral modulation.

As mentioned earlier, the dominant frequency can be decreased by an increase in the gap. Thus, for example, the same turbutron with a $1-\mathrm{cm}$ gap will produce $1 \mathrm{GW}$ in $X$ band at $10 \mathrm{GHz}$ with spectral characteristics very similar to those of the reflex triode. There is a much weaker but more complicated dependence of the location of the various spectral peaks on the gap voltage.

The power may be significantly increased by an increase in the gap voltage. Of course, to maintain the dominant frequency near $35 \mathrm{GHz}$, the gap must be increased also. Thus, for example, a voltage of $3 \mathrm{MV}$ across a $4.4-\mathrm{mm}$ gap will produce $150 \mathrm{MW} / \mathrm{sr} \cdot \mathrm{GHz}$ near $35 \mathrm{GHz}$ over a band of $\sim 3 \mathrm{GHz}$ wide and perpendicular to the beam. This is a fivefold increase in power over the prototype. The power densities at $9.5,16,22$, and $27 \mathrm{GHz}$ are also near this level. For the 3-MV gap voltage, the total power radiated in all bands is $\sim 4 \mathrm{GW} / \mathrm{sr}$. Nominally, a several gigawatt peak-power millimeter-wave source at $35 \mathrm{GHz}$ should be obtainable from a turbutron with $3 \mathrm{MV}$ across a 4.4-mm gap. At lower frequencies, for example, in $X$ band, it is likely that tens of gigawatts of peak power can be obtained from a turbutron with $\sim 3 \mathrm{MV}$ across a $\sim 1$ $\mathrm{cm}$ gap. At frequencies above $35 \mathrm{GHz}$ it is possible that the turbutron can also be a gigawatt level source. For example, with $3 \mathrm{MV}$ across a 3.4-mm gap, a turbutron will produce an average transverse power spectral density near $46 \mathrm{GHz}$ of $330 \mathrm{MW} / \mathrm{sr} \cdot \mathrm{GHz}$. Still greater high-power microwave performance characteristics for the turbutron are likely to await discovery because of the richness of its nonlinear electron dynamics.

The present one-dimensional calculations cannot include parametric dependence on the confining magneticfield strength; however, it is likely that the turbutron power spectrum will be sensitive to the applied magnetic field, as was the case with the reflex triode [10], [12].

It is also to be emphasized that the gigawatt millimeterwave power levels are obtainable from the turbutron without the aid of resonators. The possibility of including a 
quasi-optical slotted-mirror resonator positioned coaxially with the beam, or shaping the extended anode structure and tuning it to the dominant mode of the electron plasma, thereby resonantly coupling the radiation field back into the beam and amplifying the dominant mode, should also be investigated. A numerical experiment with the reflex triode, in which a 0.1-MV RF voltage signal at the dominant $10-\mathrm{GHz}$ frequency was applied across the gap during discharge, resulted in 40 -fold amplification of the power at $10 \mathrm{GHz}$ and, because of the nonlinearities, up to 240 and 400 -fold amplification at 20 and $29 \mathrm{GHz}$ [6], [7]. Analogous behavior would be expected for the turbutron. Such nonlinear mode coupling mechanisms might be used to pump power into higher-frequency bands.

Since the detailed spectral characteristics of the turbutron depend on the dynamical spectrum of the relativistic electron plasma, it is likely that the theory of electron plasma turbulence as it bears on the parametric dependence of bunching and relative phase-locking mechanisms will suggest more sophisticated methods for spectral design.

\section{CONCLUSION}

The turbutron is expected to be a viable new high-power pulsed millimeter-wave source consisting of an intensely oscillating turbulent relativistic electron plasma created between the emission and virtual cathodes of a high-voltage pulsed diode configuration under conditions of charge saturation. For a turbutron with a megavolt pulse across a 3-mm gap, calculations predict the immediate feasibility of achieving gigawatt power levels at $35 \mathrm{GHz}$. Still higherpower levels should be obtainable by means of higher voltage and/or a resonator.

\section{REFERENCES}

[1] H. E. Brandt, A. Bromborsky, S. E. Graybill, and R. A. Kehs, "The turbutron-A new high power millimeter-wave source," Bull. Amer. Phys. Soc., vol. 27, p. 1018, 1982.

[2] H. E. Brandt, A. Bromborsky, H. B. Bruns, R. A. Kehs, and G. P. Lasche, "Gigawatt microwave emission from a relativistic reflex triode," Harry Diamond Laboratories, Adelphi, MD, Rep. HDLTR-1917, Aug. 1980.

[3] R. A. MaHaffey, P. Sprangle, J. Golden, and C. Kapetanakos, "High power microwaves from a nonisochronic reflecting electron system," Phys. Rev. Lett., vol. 39, p. 843, 1977.

[4] A. N. Didenko, Y. Y. Krasik, S. F. Perelygin, and G. P. Fomenko, Pis'ma Zh. Tekn. Fiz., vol. 5, p. 321, 1979.

[5] H. E. Brandt, A. Bromborsky, H. B. Bruns, and R. A. Kehs, "Microwave radiation from relativistic electron space-charge oscillations," 1977, unpublished preprint.

[6] H. E. Brandt, A. Bromborsky, H. B. Bruns, and R. A. Kehs, "Microwave generation in the reflex triode," in Proc. 2nd Int. Topical Conf. High Power Electron. Ion Beam Res. Tech. (Ithaca, NY), Oct. 1977, vol. II, p. 649.

[7] H. E. Brandt and A. Bromborsky, "One dimensional model of relativistic Barkhausen oscillations," Bull. Amer. Phys. Soc., vol. 22, p. 1131, 1977.
[8] A. Bromborsky, H. E. Brandt, H. B. Bruns, R. Kehs, and G. Lasche, "Intense microwave emission from a relativistic reflex triode," presented at the 20th Annu. Meeting Div. Plasma Physics Amer. Phys. Soc., Colorado Springs, CO, Oct. 30-Nov. 3, 1978.

[9] H. E. Brandt, A. Bromborsky, H. B. Bruns, R. A. Kehs, and G. Lasche, "Gigawatt microwave emission from a relativistic reflex triode," Bull Amer. Phys. Soc., vol. 24, p. 1077, 1979.

[10] G. P. Lasche, A. Bromborsky, R. Kehs, M. Litz, and H. E. Brandt, "Parametric study of high power pulsed microwave emission from a relativistic reflex triode," Bull. Amer. Phys. Soc., vol. 24, p. 1070, 1979.

[11] R. A. Kehs, H. E. Brandt, and A. Bromborsky, "Electron distribution in the relativistic reflex triode," Bull. Amer. Phys. Soc., vol. 25, p. 888, 1980.

[12] R. A. Kehs, H. E. Brandt, A. Bromborsky, and G. Lasche, "The generation of gigawatt power levels of microwave radiation," presented at the Army Science Conf., West Point, NY, 1980.

[13] —, "Army tube tests promise source of millimeter power," Microwave Systems News (Review), vol. 10, p. 27, Oct. 1980.

[14] D. J. Sullivan, "High power microwave generator using relativistic electron beam in waveguide drift tube," U.S. Patent 4345 220, Aug. 17, 1982.

[15] D. J. Sullivan, "Applications of the virtual cathode in relativistic electron beams," in Proc. 3rd Int. Topical Conf. High-Power Electron Ion Beam Res. Tech. (Novosibirsk, USSR), 1979, vol. II, p. 769.

[16] D. J. Sullivan, "A high frequency vircator microwave generator," in Proc. 5th Int. Conf. High-Power Particle Beam (San Francisco, CA), 1984 , p. 557.

[17] R. D. Scarpetti, R. L. Lundberg, S. C. Burkhart, "Experimental studies of a high power pulsed microwave generator," Bull. Amer. Phys. Soc., vol. 29, p. 1180, 1984.

[18] R. R. Bartsch and H. A. Davis, "Observation of high-power microwave emission from a virtual cathode device," Bull. Amer. Phys. Soc., vol. 29, p. 1179, 1984.

[19] T. J. T. Kwan, "High-power microwave generation from oscillating virtual cathodes," Phys. Fluids, vol. 27, p. 228, 1984.

[20] T. J. T. Kwan and L. E. Thode, "Formation of virtual cathodes and microwave generation in relativistic electron beams," Phys. Fluids, vol. 27, p. 1570, 1984.

[21] C. Ekdahl, chairman; talks by C. Ekdahl, H. Davis, T. Kwan, D. Sullivan, A. Kadish, D. Voss, R. Scarpetti, and H. Brandt, presented at the Virtual-Cathode Microwave Generator Design Review, Los Alamos Nat. Lab., Los Alamos, NM, Jan. 31, 1983.

[22] O. Buneman, "Dissipation of currents in ionized media," Phys. Rev., vol. 115, p. 503, 1959.

[23] C. K. Birdsall and W. B. Bridges, "Space-charge instabilities in electron diodes and plasma converters," J. Appl. Phys., vol. 32, p. 2611, 1961.

[24] J. Dawson, "One-dimensional plasma model," Phys. Fluids, vol. 5, p. $445,1962$.

[25] C. K. Birdsall and W. B. Bridges, ', Space-charge instabilities in electron diodes, II,' J. Appl. Phys., vol. 34, p. 2946, 1963.

[26] C. K. Birdsall and W. B. Bridges, Electron Dynamics of Diode Regions. New York: Academic, 1966.

[27] J. M. Dawson, "The electrostatic sheet model for a plasma and its modification to finite-size particles," in Methods of Computational Physics, vol. 9. New York: Academic, 1970, p. 1.

[28] C. K. Birdsall, "Electron diode dynamics; Limiting currents; Plasma diodes," in Proc. Symp. Plasma Double Layers (Riso Nat. Lab., Roskilde, Denmark), June 16-18, 1982, Rep. Riso-R-472, p. 84.

[29] V. N. Tsytovich, Theory of Turbulent Plasma. New York: Plenum, 1977.

[30] J. P. Eckmann, "Roads to turbulence in dissipative dynamical systems," Rev. Mod. Phys., vol. 53, p. 643, 1981.

[31] E. Ott, "Strange attractors and chaotic motions of dynamical systems," Rev. Mod. Phys., vol. 53, p. 655, 1981.

[32] R. H. G. Helleman, Ed., Nonlinear Dynamics, vol. 357. New York: New York Academy of Sciences, 1980.

[33] D. Campbell and H. Rose, Eds., "Order in chaos," Physica D, vol. 7D, pp. 1-361, 1983. 